\title{
Community pharmacists' perceptions of acceptability, appropriateness, and feasibility of a value-based care model for comprehensive medication management
}

\author{
Deborah L Pestka, PharmD, PhD; Morgan K Stoa, PharmD; Todd D Sorensen, PharmD, FCCP; \\ and Carrie M Blanchard, PharmD, MPH
}

\section{What is already known about this subject}

- Comprehensive medication management (CMM) is necessary to address the burden of suboptimal medication use, yet the uptake of CMM has been sporadic.

- Implementation science and the study of implementation outcomes is essential to accelerate the adoption of CMM.

\author{
What this study adds \\ - Generally, community pharmacists \\ and pharmacy managers have positive \\ perceptions of the acceptability, \\ appropriateness, and feasibility of a \\ regional health plan's value-based \\ care model, yet there are some \\ implementation barriers that still exist. \\ - Study results shed light on how a \\ value-based care model is perceived \\ within community pharmacies and \\ could inform the development and \\ implementation of similar CMM \\ programs.
}

\author{
Author affiliations \\ Deborah L Pestka, PharmD, PhD; \\ Morgan K Stoa, PharmD; and Todd D \\ Sorensen, PharmD, FCCP, Department of \\ Pharmaceutical Care \& Health Systems, \\ College of Pharmacy, University of \\ Minnesota, Minneapolis. Carrie M Blanchard, \\ PharmD, MPH, Center for Medication \\ Optimization, UNC Eshelman School of \\ Pharmacy, University of North Carolina at \\ Chapel Hill.
}

AUTHOR CORRESPONDENCE: Deborah L Pestka, 612.626.9938; pestk003@umn.edu

J Manag Care Spec Pharm. 2021;27(7):865-72

Copyright $\odot 2021$, Academy of Managed Care Pharmacy. All rights reserved.

outcomes of acceptability, appropriateness, and feasibility.

provides key insights into the uptake of patient care services, such as CMM, into practice.

OBJECTIVE: To evaluate the acceptability, appropriateness, and feasibility of the PIE program from the perspective of community pharmacists and pharmacy managers.

METHODS: Semi-structured, one-on-one qualitative interviews were conducted with a group of 14 pharmacists and pharmacy managers participating in the PIE program. Interviews were coded inductively, and then codes were mapped to the implementation
RESULTS: Twelve codes emerged from the interviews. Four codes (targeted conditions of PIE, achieving PIE metrics, comprehensiveness of PIE, and confusion and barriers) were mapped to acceptability; 3 codes (CMM documentation and billing, fitting CMM into limited time with patients, and community pharmacy's role in patient care) were mapped to appropriateness; and 1 code (collecting clinical patient information) was mapped to feasibility. Four codes (CMM 
payment model, targeting patients for CMM, personnel for CMM, and patient/provider buy-in of CMM) were considered a combination of more than 1 outcome.

CONCLUSIONS: Although the acceptability, appropriateness, and feasibility of the PIE program was generally positive, participants cited a number of implementation challenges related to documentation and billing and producing a sustainable CMM model. The results shed light on how a value-based care model for CMM is perceived within community pharmacies and could inform the development and implementation of similar quality-based CMM programs.

The burden of suboptimal medication use in US health care has been well documented. ${ }^{1}$ Medication management services by pharmacists have long been recognized as a strategy to address this burden, and a number of studies have demonstrated a positive impact on clinical and cost variables..$^{2-4}$ Despite the growing recognition of preventable drug-related morbidity and decades-long conversations within the profession of pharmacy regarding the need to adopt new patient care models to address this public health problem, the presence of comprehensive clinical services in community pharmacies remains sporadic.

A reason commonly cited for this reality is the lack of compensation for clinical services by pharmacists, separate from payment for drug distribution activities. ${ }^{5}$ In scenarios where clinical service payment has been offered, pharmacist engagement is often limited. For example, Minnesota Medicaid adopted payment for comprehensive medication management (CMM) in 2006. In 2011, an analysis of CMM claims submitted by pharmacists demonstrated that only $3.5 \%$ of over 37,000 eligible Medicaid beneficiaries had a CMM encounter as documented by the presence of a CMM claim during that year. Of the 110 pharmacy organizations that submitted claims, fewer than $5 \%$ were submitted by pharmacists practicing in community pharmacies. ${ }^{6} \mathrm{~A}$ review of the Nova Scotia Pharmacare Program also demonstrated limited community pharmacist engagement in a program offering compensation for clinical service delivery by pharmacists.

For health care payers and pharmacy leaders who wish to connect medication management services provided by pharmacists in the community pharmacy setting to the opportunity to improve public health and reduce health care costs, it is important to recognize that payment for services is not the only variable that will drive adoption of service delivery. Compensation is one enabling factor that can support a change in patient care delivery. In order to produce wide-scale change that can replicate the outcomes recognized in previous evaluations, a more comprehensive look into the implementation of CMM services is needed. It is important to note that CMM is frequently used interchangeably with medication therapy management (MTM). However, MTM often refers to Medicare's MTM program, which has defined patient inclusion criteria and program requirements. CMM, on the other hand, can be delivered to any patient with potential for medication therapy problems and can be offered as frequently as necessary to get patients to their desired goals.

Implementation science is the study of the "systematic uptake of research findings and other evidence-based practices into routine practice." Important to implementation science is the study of implementation outcomes. Proctor et al. identified 8 implementation outcomes to monitor progress of an intervention and successful practice change: adoption, acceptability, appropriateness, cost, feasibility, fidelity, penetration, and sustainability. ${ }^{9}$ Choosing which implementation outcome to measure depends on the stage of implementation, the research question of interest, and relevance to the stakeholder. ${ }^{10}$

Early implementation outcomes, such as acceptability, appropriateness, and feasibility, serve as key intermediate outcomes that provide important information on how patient care transformation efforts are going. Acceptability is defined as the different stakeholders' perceptions that a new service model is agreeable, palatable, or satisfactory. Appropriateness reflects the perceived fit, relevance, or compatibility of a service for a given practice setting (eg, community pharmacy) and patient populations. The extent to which a new service can be successfully used or carried out within a given setting is a measure of feasibility. ${ }^{9}$

Many times, evidence-based practices do not succeed in real-world settings because of competing demands, lack of knowledge and skills, and other implementation barriers. ${ }^{11}$ Therefore, when an intervention such as CMM is being implemented, it is important to evaluate health outcomes, such as effectiveness and cost, along with implementation outcomes.

In 2010, HealthPartners, a regional health plan based in Bloomington, Minnesota, introduced a value-based care model for CMM services for its commercially insured members. In 2015, the program was expanded to include a performance-based component titled Partners in Excellence (PIE). However, participation by pharmacists practicing in community pharmacies has been limited. Thus, the objective of this study was to evaluate the acceptability, appropriateness, and feasibility implementation outcomes of HealthPartner's value-based care model for CMM services from the perspective of community pharmacists and managers. 


\begin{tabular}{l|c}
\multicolumn{1}{c|}{ TABLE 1 } & $\begin{array}{l}\text { Participant } \\
\text { Characteristics }\end{array}$ \\
\hline \multicolumn{1}{|c}{ Characteristic } & Number \\
\hline Fex & 9 \\
\hline Male & 5 \\
\hline ge, years, $\mathrm{n}=12$ & \\
\hline 25-34 & 4 \\
\hline 35-44 & 3 \\
\hline 45-54 & 3 \\
\hline 55-64 & 2 \\
\hline $\begin{array}{l}\text { Years spent working at current } \\
\text { harmacy organization, }\end{array}$ & $11(9.8)$ \\
mean (SD), n=12 & \\
\hline
\end{tabular}

\section{Methods}

\section{PIE PROGRAM}

The HealthPartners PIE program recognizes and rewards pharmacy practices that improve patient health and engagement through CMM. HealthPartners' expectations for these pharmacists is that they provide care in alignment with the patient care process for CMM. ${ }^{12}$ The health plan provides quarterly registries to participating pharmacies of patients deemed at risk for medication-related problems based on available medical and prescription claims data. Compensation for services is provided via a combination of encounter-based payment (fee-for-service), as well as an annual performance-based (ie, achievement of patient outcomes) payment strategy.

Starting in 2019, HealthPartners instituted quality and engagement metrics as part of the PIE program. Performance benchmarks are set for 3 clinical outcome targets in patients with diabetes and vascular conditions: (1) blood pressure goal <140/90; (2) tobacco-free status; and (3) hemoglobin A1c rates less than $8 \%$ in patients with diabetes.
There is also an engagement metric if organizations in the HealthPartners CMM network provide CMM services to $40 \%$ or more of eligible patients engaging in CMM by the end of each program year. Practices then receive bonus payments based on their achievement of the clinical and engagement metrics. While these are the target measures put forth by HealthPartners, it is expected that the pharmacists provide a comprehensive visit assessing all medications and conditions for each patient.

\section{SLICE OF PIE}

As a subset of the larger PIE program, Slice of PIE is an 18-month implementation and research initiative that began in September 2019 with a partnership that includes HealthPartners, the University of Minnesota, and the University of North Carolina. Slice of PIE focuses on building community pharmacist engagement in the PIE program to help pharmacies achieve the patient engagement and clinical performance goals set forth by the PIE program. All pharmacies within the HealthPartners CMM network were invited to participate in Slice of PIE. Twelve organizations and 45 pharmacists within those organizations signed up to participate in Slice of PIE. Six of the pharmacies were independent pharmacies, while the remaining 6 were pharmacies having 3 or more regional locations.

\section{STUDY DESIGN AND PARTICIPANTS}

To obtain the perspectives of management and pharmacists, CMM managers and pharmacists were invited to participate in qualitative interviews regarding their perspectives on the acceptability, appropriateness, and feasibility of the PIE program. Qualitative interviews provide an opportunity to obtain rich descriptions from participants that cannot easily be achieved through other methods. To maximize participation, interviews were selected over focus groups so that interviewees could select a time to be interviewed that was most convenient for them. CMM managers were purposefully selected, whereas pharmacists were randomly selected. In total, 4 CMM managers and 10 pharmacists agreed to participate in the interviews (Table 1). At least 1 pharmacist or manager from each of the 12 participating organizations participated in an interview.

\section{DATA COLLECTION}

Participants were sent an email in December 2019 requesting participation in a 30-minute interview via phone. Interviews were conducted December 20, 2019-January 22, 2020, and were audio recorded and transcribed verbatim. An interview guide was developed to understand participants' perceptions of the PIE program in terms of the implementation outcomes of acceptability, appropriateness, and feasibility (Supplementary File 1, available in online article). The interview guide was developed with feedback from the research team, as well as 2 community pharmacists providing CMM. The University of Minnesota Institutional Review Board determined that this study did not need to undergo formal review.

\section{DATA ANALYSIS}

Interview transcripts were coded by a member of the research team using an inductive approach, meaning that codes were not developed a priori, but rather were developed based on what occurred in the transcripts. The initial codebook with illustrative quotes was shared with the larger research team for feedback and input. In addition, a second member of the research team reviewed 6 randomly selected transcripts to ensure that codes and definitions were appropriate. The 


\begin{tabular}{|c|c|c|}
\hline Outcome & Proctor et al definition 9 & Application to the PIE program \\
\hline Acceptability & $\begin{array}{l}\text { "The perception among implementation stakeholders } \\
\text { that a given treatment, service, practice, or innovation is } \\
\text { agreeable, palatable, or satisfactory." }\end{array}$ & $\begin{array}{l}\text { Pharmacy managers' and pharmacists' thoughts related } \\
\text { specifically to the PIE program. }\end{array}$ \\
\hline Appropriateness & $\begin{array}{l}\text { "The perceived fit, relevance, or compatibility of the } \\
\text { innovation or evidence-based practice for a given practice } \\
\text { setting, provider, or consumer; and/or perceived fit of the } \\
\text { innovation to address a particular issue or problem." }\end{array}$ & $\begin{array}{l}\text { Pharmacy managers' and pharmacists' thoughts about the } \\
\text { fit of the PIE program in their pharmacies/organizations, } \\
\text { including motivation for delivering CMM. }\end{array}$ \\
\hline Feasibility & $\begin{array}{l}\text { "The extent to which a new treatment, or an innovation, } \\
\text { can be successfully used or carried out within a given } \\
\text { agency or setting." }\end{array}$ & $\begin{array}{l}\text { Pharmacy managers' and pharmacists' ability (or lack } \\
\text { thereof) to successfully carry out the PIE program in their } \\
\text { pharmacies and/or organizations. }\end{array}$ \\
\hline
\end{tabular}

$C M M=$ comprehensive medication management; $P I E=$ Partners in Excellence

2 coders then met to discuss any modifications that needed to be made to the codebook, and the final codebook was applied to all transcripts. To enhance validity of the data, a summary of codes and definitions were sent to interview participants for review and feedback as a form of member checking.

To group the identified codes to implementation outcomes, the research team defined the implementation outcomes in the context of the PIE program (Table 2), and each member of the team independently linked each code to 1 of the implementation outcomes. The research team then met to compile responses and discuss differences. Where there was disagreement, the definitions of the implementation outcomes were discussed and modified until consensus was reached.

\section{Results}

Twelve codes emerged from the interviews that were mapped to the implementation outcomes (Figure 1). Four codes were mapped to acceptability, 3 to appropriateness, and 1 to feasibility, and 4 codes were considered a combination of more than 1 outcome. Supplementary Table 2 (available in online article) displays the identified codes, as well as an illustrative quote for each code. The following sections describe each code (indicated in italics) under the implementation outcome to which it was mapped.

\section{ACCEPTABILITY}

Pharmacists and managers were in general agreement that the target conditions of the PIE program (diabetes, hypertension, and smoking cessation) were conditions that matched the needs of their patient population and that they were in alignment with conditions used for national quality measures, such as the Medicare star ratings system. However, there was some hesitation about smoking cessation because it was cited as a topic that pharmacists were less comfortable discussing with patients, and it was difficult to engage patients. Additionally, there was some desire to expand to target patients for the PIE program beyond those conditions.

While there were comments that certain PIE metrics could be higher or lower, participants were in general agreement with the metrics of the PIE program. Furthermore, a pharmacist and manager commented that, although $40 \%$ engagement seemed like a low goal, it was actually a challenge to achieve. Five participants also commented that they liked how comprehensive the PIE program was compared with other CMM programs. Three pharmacists commented that they felt they were providing better CMM due to the higher expectations of the PIE program. Finally, there was some confusion and barriers that participants mentioned related to the PIE program. For example, some discussed barriers to billing through the HealthPartners online portal and delivering CMM longitudinally to patients when they frequently change insurers.

\section{APPROPRIATENESS}

With regard to documentation and billing, HealthPartners allows pharmacies to use a CMM documentation system of their choosing. However, 3 managers mentioned that the costs of some of these nondispensing-related systems can prohibit their participation in PIE. There was also concern about having the knowledge to submit claims correctly, that the billing process was more tedious compared with other programs, and that there was duplicate work occurring, since clinical documentation and billing could not be done in the same system. While not specific to PIE, some expressed a desire for documentation platforms to better 


\section{FIGURE 1 Mapping of Qualitive Codes to Implementation Outcomes}

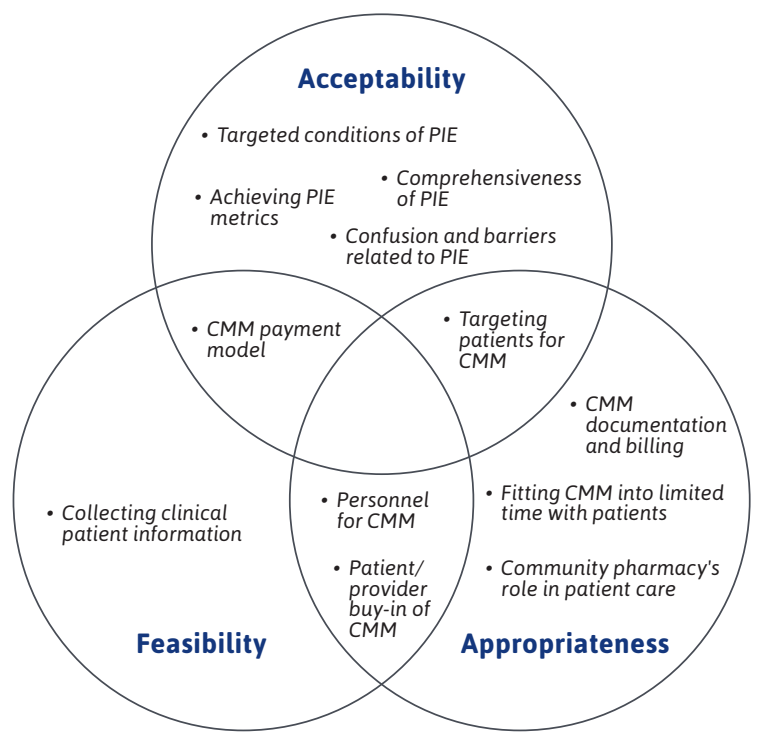

$C M M=$ comprehensive medication management; PIE = Partners in Excellence.

integrate with pharmacy systems and the desire for documentation to be integrated with electronic health records.

Participants felt that the PIE program appropriately fit with their belief that community pharmacists provide patient care. Nine participants felt that community pharmacies should be involved in more patient care. In addition, a couple of independent pharmacists mentioned that they felt being a smaller pharmacy provided an advantage to delivering patient care. However, there were some competing priorities and issues regarding resources that many mentioned as challenges to fitting CMM into limited time with patients. For example, having less time during flu season and decreased staff resources were mentioned as things that limit CMM completion.

\section{FEASIBILITY}

With regards to collecting clinical patient information (eg, A1c and blood pressure) that is needed for CMM visits within the PIE program, participants had a range of thoughts on the feasibility of obtaining such information. For example, some pharmacists and managers stated that they can collect this information in their pharmacies. However, others rely on patient reporting or contacting the patient's clinic. There were a number of challenges stated with collecting patient information, such as patients not knowing their lab values, difficulty in contacting clinics, and the need to have a release if obtaining information from the patient's clinic.

\section{OVERLAPPING OUTCOMES}

There were a number of codes that overlapped with implementation outcomes. For example, achieving patient and provider buy-in for CMM and personnel for CMM contained aspects of appropriateness and feasibility. One of the challenges of participating in a program such as PIE is the patient and provider buy-in that is needed. Three pharmacists discussed the difficulty of reaching out to patients when they have not been informed about the service or may not know the pharmacist who is calling them. Similarly, 2 pharmacists mentioned how it can be challenging to change patients' medications when doing so relies on the responsiveness of medical providers because some medical providers' offices may take a long time to respond to requests or requests may not be seen by providers. On the other hand, a manager working in a pharmacy that was located within a clinic noted how being in the same building as the providers has significantly increased their buy-in.

For the personnel to deliver CMM, 9 participants discussed engaging pharmacy technicians, students, and residents in the process by having them do various tasks such as educating patients about the program, collecting some patient history such as immunizations, and scheduling encounters. However, personnel varied from pharmacy to pharmacy as did the pharmacists' comfort level with engaging various types of pharmacy staff in CMM. Also, not all pharmacies consistently have residents and/or students, and some managers felt certain pharmacists were more interested in being involved with CMM than others.

Targeting patients for CMM included elements of acceptability and appropriateness. With regards to the patients who are targeted for the PIE program, there was a wide range in the number of patients that pharmacies received through HealthPartners CMM registries. Additionally, there was variation in how many CMM encounters pharmacists thought they could provide (eg, 1 pharmacist suggested 2 per week, while another suggested 2-3 per day).

In the PIE program, pharmacies may self-identify patients beyond the registry of patients they are provided (specifically, patients who have a diagnosis of diabetes or cardiovascular disease), and 1 manager commented that he liked this aspect because he did not always feel that algorithms used to target patients are accurate. On the other hand, another manager commented that she feels that selfidentifying requires a lot of resources for something that should be done by the health plan. In general, participants felt that patients targeted for CMM through PIE were more 
complicated compared with patients seen through other platforms.

The CMM payment model overlapped with acceptability and feasibility. There were differing opinions on the financial incentives offered through the PIE program. One pharmacist indicated that money does not provide motivation to be involved in a program such as PIE. On the other hand, several participants mentioned that incentives are good, but they would like to see higher reimbursement. Finally, 2 managers suggested a move to a per-member-permonth model.

\section{Discussion}

This study evaluated how community pharmacy managers and pharmacists perceived the acceptability, appropriateness, and feasibility of a value-based care model for CMM. For the most part, participants felt positively about these implementation outcomes. However, there were barriers cited by some within certain codes. For example, a number of interviewees spoke of the difficulties with care documentation and submitting claims for service delivery. While the PIE program does not require use of any specific clinical documentation or claims submission technology, participants cited challenges with the general cost of documentation platforms, different platforms required by different CMM programs (eg, Outcomes and Tabula Rasa), and the challenge of correctly submitting claims. Having multiple documentation systems and a lack of interoperability between documentation platforms or the presence of a single platform is an issue that has previously been documented as a barrier to the delivery of CMM. ${ }^{13,14}$

Furthermore, billing for patient care services can be complex and difficult to navigate for many pharmacists..$^{15,16}$ Therefore, even in a unique value-based care model for CMM where pharmacies are given the choice of which documentation system to use, issues related to documentation and claims still persist.

A common theme from the interviews was the payment model for PIE. Health care is increasingly shifting away from traditional fee-for-service payment models and towards value-based payment. In line with this, a few managers suggested changing the financial structure of the PIE program to a value-based, per-member-per-month payment structure. Previous studies on CMM have demonstrated a 12:1 return on investment. ${ }^{17}$ These significant cost savings could allow for health plans to share the savings acquired from delivery of CMM services with the community pharmacies delivering the service. Models that leverage these savings may increase the public health impact of CMM by stimulating more community pharmacies to engage in CMM services and may serve to be a more financially sustainable model.

The PIE program is an example of a payer-provider partnership because the payer, HealthPartners, is committed to supporting pharmacists in their efforts to provide CMM. A number of codes identified affected participants' success in PIE but are applicable to CMM delivered in community pharmacies in general. For example, some participants spoke of challenges of fitting CMM into pharmacy workflows, engaging pharmacy personnel in CMM, patient and provider buy-in of CMM, and collecting clinical patient information. These types of issues have long been cited as barriers to CMM. ${ }^{5,18}$ To overcome these challenges and ensure widespread access to CMM for patients, it is important that community pharmacy leadership ensure that pharmacists have dedicated time to provide patient care tasks and necessary support structures, such as a documentation platform and technician support, if necessary.

Likewise, the results of this study suggest that there are actions payers could take to further support a payerprovider partnership. For example, payers could explore options to provide pharmacies with the clinical information they need to provide optimal CMM. In addition, payers could support patient and provider buy-in by educating patients and providers on the public health value of CMM and notifying them that a pharmacist may be contacting them regarding CMM.

Payer-provider partnerships should consider the role of investing in formal implementation support structures. While not the focus of this study, the Slice of PIE program includes several implementation supports such as facilitation of a learning community among the pharmacist participants, monthly coaching activities focused on practice transformation, hosted learning and sharing events, and availability of educational modules at no cost to the participants.

There is a public health need for broader patient access to CMM, and value-based care models may provide an opportunity to address this need. It is important to assess the implementation strategies of acceptability, appropriateness, and feasibility in the early stages of implementation, since these outcomes affect other implementation outcomes, such as fidelity, adoption, and penetration. ${ }^{9}$ The results of this work suggest that there are some promising features of a value-based care model, such as PIE. However, to accelerate implementation, pharmacists and pharmacies must be able to commit the time and staffing to CMM, and payers must be able to provide implementation supports for CMM services. 


\section{LIMITATIONS}

This study has some limitations to consider. One limitation is that all pharmacies were located in Minnesota. CMM has been delivered within certain health systems and pharmacies within Minnesota for over 20 years, which may have influenced participants perceptions of the implementation of the PIE program. In addition, there were no large pharmacy chains involved in this project, only independent and regional chains. Therefore, these findings may not be generalizable to pharmacists practicing in larger organizations. Also, pharmacies and pharmacists were at different stages of their knowledge and adoption of CMM. Some were in the early stages of providing CMM services, while others had been delivering this service for years. Therefore, different levels of exposure to CMM may have affected their perceptions of these implementation outcomes.

\section{Conclusions}

As pharmacy evolves and the public health need to optimize medications intensifies, it is critical for pharmacists to adopt and implement CMM services within their organizations. The results of this study shed light on how a program such as PIE is perceived within community pharmacies. It is important to assess acceptability, appropriateness, and feasibility early when implementing new care models, such as the PIE program, since these outcomes can affect implementation success. The results of this assessment are important for the continual development of strategies to maximize CMM engagement and optimize medication outcomes and access to CMM for patients. The results could inform the development and implementation of similar CMM value-based care programs.

\section{DISCLOSURES}

This study was funded by the National Association of Chain Drug Stores (NACDS) Foundation and the UNC Eshelman Institute for Innovation. Pestka is affiliated with the University of Minnesota College of Pharmacy and reports grants from NACDS Foundation and UNC Eshelman Institute for Innovation for the conduct of the study; she has also received grants from UNC Eshelman Institute for Innovation and NACDS Foundation outside the submitted work. Stoa and Sorensen are also affiliated with the University of Minnesota College of Pharmacy. Blanchard is employed at the UNC Eshelman School of Pharmacy, University of North Carolina at Chapel Hill.

This work was presented as a virtual poster at the 2020 American College of Clinical Pharmacy Annual Meeting, October 19-30, 2020 .

\section{REFERENCES}

1. Watanabe JH, McInnis T, Hirsch JD. cost of prescription drug-related morbidity and mortality. Ann Pharmacother. 2018;52(9):829-37.

2. Gazda NP, Berenbrok LA, Ferreri SP. Comparison of two medication therapy management practice models on return on investment. J Pharm Pract. 2017;30(3):282-85.

3. Brummel AR, Soliman AM, Carlson AM, de Oliveira DR. Optimal diabetes care outcomes following face-to-face medication therapy management services. Popul Health Manag. 2013;16(1):28-34.

4. Budlong H, Brummel A, Rhodes A, Nici H. Impact of comprehensive medication management on hospital readmission rates. Popul Health Manag. 2018;21(5):395-400.

5. Lounsbery JL, Green CG, Bennett MS Pedersen CA. Evaluation of pharmacists' barriers to the implementation of medication therapy management services. J Am Pharm Assoc (2003). 2009;49(1):51-58.

6. Wallace M, Drake S. MN Medicaid MTM claims 2008-2014. Oral presentation at: Mobilizing Practice Transformation in Medication Management for the Triple Aim. 2014; Minneapolis, MN.
7. Deal HJ, Cooke CA, Ingram EML, Sketris IS. Adoption of the Nova Scotia (Canada) community pharmacy medication management program, 1-year post-initiation. J Popul Ther Clin Pharmacol. 2017;24(1):e46-e55.

8. Eccles MP, Mittman BS. Welcome to implementation science. Implement Sci. 2006;1(1):1.

9. Proctor E, Silmere H, Raghavan R, et al. Outcomes for implementation research: conceptual distinctions, measurement challenges, and research agenda. Adm Policy Ment Health. 2011;38(2):65-76.

10. Livet M, Haines ST, Curran GM, et al. Implementation science to advance care delivery: a primer for pharmacists and other health professionals. Pharmacotherapy. 2018;38(5):490-502.

11. Bauer MS, Damschroder L, Hagedorn H, Smith J, Kilbourne AM. An introduction to implementation science for the nonspecialist. BMC Psychol. 2015;3(1):32.

12. McClurg MR, Sorensen TD, Carroll J. The patient care process for delivering comprehensive medication management (CMM): optimizing medication use in patient-centered, team-based care settings. July 2018. Accessed September 23, 2020. https://www.accp.com/docs/positions/misc/CMM_Care_Process.pdf

13. Shoemaker SJ, Hassol A. Understanding the landscape of MTM programs for Medicare. Part D: results from a study for the Centers for Medicare \& Medicaid services. J Am Pharm Assoc (2003). 2011;51(4):520-26.

14. Millonig MK. Mapping the route to medication therapy management documentation and billing standardization and interoperabilility within the health care system: meeting proceedings. J Am Pharm Assoc (2003). 2009;49(3):372-82.

15. Law AV, Okamoto MP, Brock K. Ready, willing, and able to provide MTM services? A survey of community pharmacists in the USA. Res Soc Adm Pharm. 2009;5(4):376-81.

16. Kliethermes MA. Understanding health care billing basics. Pharm Today. 2017;23(7):57-68. 
17. Brummel A, Lustig A, Westrich K, et al. Best practices: improving patient outcomes and costs in an ACO through comprehensive medication therapy management. J Manag Care Spec Pharm. 2014;20(12):1152-158. doi: 10.18553/

jmcp.2014.20.12.1152
18. American Pharmacists Association. Medication therapy management services: developing a practice as an independent MTM pharmacist. 2008. Accessed June 8, 2021. https://aphanet.pharmacist. com/sites/default/files/files/mtm developing a indie practice.pdf 